anticoagulation alone in some cases, we believe that the embolic potential of a mobile ascending aortic thrombus warrants prompt intervention.

\footnotetext{
References

1. Reber PU, Patel AG, Stauffer E, Muller WF, Do DD, Kniemeyer HW. Mural aortic thrombi: an important cause of peripheral embolization. JVasc Surg. 1999;30:1084-9.

2. Blackshear JL, Jahangir A, Oldenburg WA, Safford RE. Digital embolization from plaque-related thrombus in the thoracic aorta: identification with transesophageal
}

echocardiography and resolution with warfarin therapy. Mayo Clin Proc. 1993; 68:268-72.

3. Hausmann D, Gulba D, Bargheer K, Niedermeyer J, Comess KA, Daniel WG. Successful thrombolysis of an aortic-arch thrombus in a patient after mesenteric embolism. N Engl J Med. 1992;327:500-1.

4. Choukroun EM, Labrousse LM, Madonnal FP, Deville C. Mobile thrombus of the thoracic aorta: diagnosis and treatment in 9 cases. Ann Vasc Surg. 2002;16: 714-22.

5. Madershahian N, Kuhn-Regnier F, Ben Mime L, Slottosch I, Langebartels G, Sindhu D, et al. A loose cannon: free-floating thrombus in ascending aorta. $J$ Card Surg. 2008;24:198-9.

\title{
Mechanical left ventricular unloading to prevent recurrent myocardial rupture
}

\author{
Stephen Westaby, MS, PhD, ${ }^{\mathrm{a}}$ Vipin Mehta, MD, ${ }^{\mathrm{a}}$ Fidelma Flynn, MD, ${ }^{\mathrm{b}}$ and Neil Wilson, $\mathrm{MD},{ }^{\mathrm{c}}$ Oxford, \\ United Kingdom
}

Temporary left ventricular assist devices (LVADs) are used to sustain systemic blood flow during acute left ventricular (LV) failure. Ventricular unloading also reduces intracavity pressure and wall tension. Decreased LV work might then allow functional recovery after postischemic stunning or viral myocarditis. ${ }^{1}$ Extrapolating from this, LV unloading could be used to protect against myocardial rupture in injured hearts with normal contractility. This strategy is illustrated by the following case.

\section{CLINICAL SUMMARY}

An otherwise healthy 15-year-old (44-kg) girl presented with a 3-week febrile illness and large pericardial effusion. Blood cultures grew Staphylococcus aureus. During hospital investigation, she experienced sudden cardiovascular collapse and pulmonary edema. Echocardiographic analysis showed severe mitral regurgitation, with large mobile vegetations filling the small left atrium. Requiring intermittent cardiac massage, she was bought to the operating room (OR). Through a median sternotomy, the $800-\mathrm{mL}$ bloodstained effusion was drained, and cardiopulmonary bypass was established. The left atrium was approached by means

\footnotetext{
From the Department of Cardiac Surgery, ${ }^{a}$ Nuffield Department of Anaesthetics, ${ }^{b}$ and Department of Paediatric Cardiology, Oxford Children's Hospital, ${ }^{\mathrm{c}}$ John Radcliffe Hospital, Oxford, Untied Kingdom.

This mechanical circulatory support programme is supported by the Gyde Trust.

Disclosures: None.

Received for publication June 17, 2009; accepted for publication July 5, 2009; available ahead of print Aug 31, 2009

Address for reprints: Stephen Westaby, MS, PhD, Department of Cardiothoracic Surgery, John Radcliffe Hospital, Headley Way, Headington, Oxford OX3 9DU, United Kingdom (E-mail: swestaby@ahf.org.uk)

J Thorac Cardiovasc Surg 2010;140:e16-7

$0022-5223 / \$ 36.00$

Copyright (c) 2010 by The American Association for Thoracic Surgery doi:10.1016/j.jtcvs.2009.07.009
}

of superoseptal incision. A 4-cm $\times 2$-cm pedunculated vegetation was removed, revealing dehiscence of the posterior mitral commissure with flail anterior and posterior leaflets. Beneath and separate from the annulus, there was ulceration of the endocardium with vegetations. The mitral valve was repaired by excising infected tissue and sewing an elliptical bovine pericardial patch into the annulus. The flail leaflets were then reattached to the patch. Vegetations were curetted from the infected LV myocardium. Intraoperative transesophageal echocardiographic analysis showed the reconstructed valve to be fully competent. Apart from temporary left-sided weakness, she made a straightforward recovery but had repeated febrile episodes.

On the seventh postoperative day, she again experienced sudden cardiovascular collapse with cardiac tamponade. On this occasion, echocardiographic analysis showed a tense posterior intrapericardial collection and an aneurysmal area at the site of the myocardial abscess. The repaired valve and mitral annulus remained intact (Figure 1). She was rapidly conveyed to the OR and started on cardiopulmonary bypass. Lifting the heart, a ruptured mycotic aneurysm of the left ventricle was identified between the circumflex marginal and distal circumflex branches. The disintegrating epicardium was debrided and closed with deep Teflon plegeted mattress sutures, avoiding the coronary branches. The following day during extubation, the ventricle ruptured again. Back in the OR, renewed attempts to close the leak occluded the dominant circumflex coronary, causing acute LV failure. Removal of the sutures restored contractility, but subsequent repair remained tenuous. To prevent further dehiscence, we used a centrifugal blood pump (Rotaflow, Maquet, Germany) to empty the left heart and reduce systolic LV wall tension. By using left atrial and ascending aortic cannulation, the LVAD was left in situ for 16 days pending control 


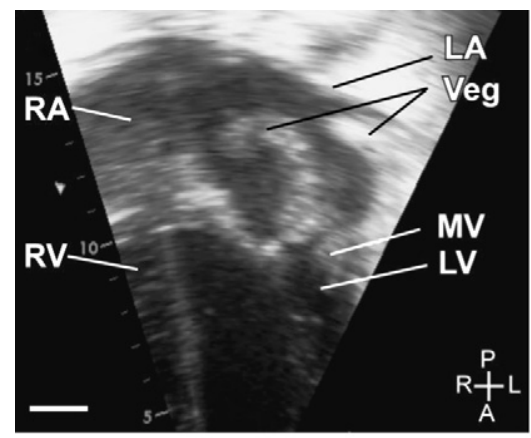

A

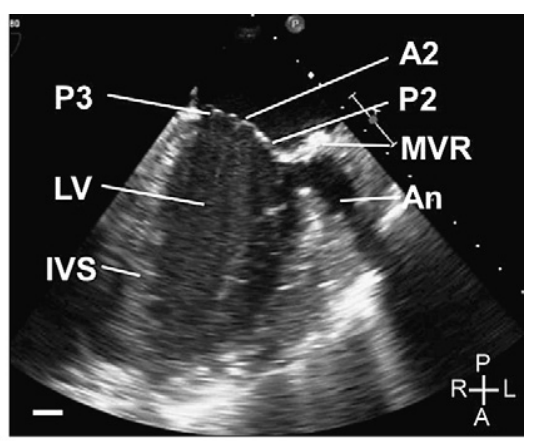

B

FIGURE 1. A, Transthoracic echocardiographic apical 2-chamber view focusing on the left atrium. There is a large vegetation (Veg) attached to the mitral valve $(M V)$ leaflets and extending into the left atrium $(L A)$. There is also extensive vegetation on the posterior left atrial wall and posterior leaflet of the mitral valve. $R A$, Right atrium; $R V$, right ventricle; $L V$, left ventricle. B, Two-chamber left ventricular and left atrial transesophageal echocardiogram view $\left(75^{\circ}\right)$. An aneurysm in the left ventricle $(L V)$ resulting in left ventricular rupture is shown. The repair of the posterior leaflet of the mitral valve with bovine pericardium is also shown $(M V R)$. The anterior and posterior segments of the mitral valve seen are indicated (P3, $A 2$, and $P 2)$. IVS, Interventricular septum; An, mycotic aneurysm. Scale bar $=1 \mathrm{~cm}$.

of infection, development of fibrosis within the aneurysmal wall, and inflammatory obliteration of the posterior pericardium. Cannulation was guided by transesophageal echocardiography so that the inflow cannulas could not impinge on the repaired valve. LVAD flow varied between 3.5 and 4.5 $\mathrm{L} / \mathrm{min}$, with a mean systemic pressure of $60 \mathrm{~mm} \mathrm{Hg}$. After closing the chest, the support period proved uneventful, as was weaning and decannulation. Blood pressure control was continued with angiotensin-converting enzyme inhibition and $\beta$-blockade to maintain systolic pressure at less than $100 \mathrm{~mm} \mathrm{Hg}$. Despite the protracted infective illness and 4 cardiac operations, subsequent recovery was uneventful. On discharge 6 weeks after decannulation, echocardiographic analysis showed a competent mitral valve with well-preserved LV function. No cause was found for the staphylococcal septicemia.

\section{DISCUSSION}

Although aortic root abscesses commonly occur in endocarditis, septic full-thickness erosion of ventricular myocardium is exceptionally rare and difficult to manage in a small heart from a female patient. ${ }^{2,3}$ The infected muscle disintegrated, and the coronary arteries were obscured by fibrin from the septic pericardial effusion. The ventricular unloading balanced potential thromboembolic or bleeding risks of circulatory support versus the benefit of reduced wall tension to prevent further LV rupture. Mitral valve repair proved durable and avoided sheer stress generated by a mechanical prosthesis at the atrioventricular junction. ${ }^{4}$

LVAD risks have substantially reduced with modern centrifugal pumps. This greatly increases the scope for their use to contain risk in cardiac surgery. ${ }^{5}$ Most effective LV unloading is normally achieved through the apex. ${ }^{5}$ On this occasion, we wished to obliterate the posterior pericardium by means of adhesion formation and could not risk impingement of an inflow cannula on the mycotic aneurysm. Pharmacologic reduction of afterload improved LVAD flow while reducing LV wall tension. This allowed healing at the site of repair.

Although myocardial infection is unusual, myocardial infarction with ventricular septal defect or free wall rupture can pose similar problems. We now have experience with LV unloading to prevent breakdown of a fragile repair in these patients.

\section{References}

1. Westaby S, Balacumaraswami L, Sayeed R. Maximising survival potential in very high risk cardiac surgery. Heart Fail Clin. 2007;3:159-80.

2. Citak M, Rees A, Mavroudis C. Surgical management of infective endocarditis in children. Ann Thorac Surg. 1992;54:755-60.

3. Beare MS. Mycotic aneurysm of the left ventricle. Thorax. 1967;22:70-2.

4. Lee EM, Shapiro LM, Wells FC. Conservative operation for infective endocarditis of the mitral valve. Ann Thorac Surg. 1998;65:1087-92.

5. Westaby S, Balacumaraswami L, Bertoni GB, Robson D, Grebenik K. Elective transfer from cardiopulmonary bypass to centrifugal blood support in very high risk cardiac surgery. Heart Fail Clin. 2007;3:159-80. 